\title{
Comparing Non-homomorphic and Homomorphic Wavelet Filtering Techniques for Speckled Images
}

\author{
M. A. Gungor and I. Karagoz
}

\begin{abstract}
Speckle is a multiplicative noise which decreases the quality of an ultrasound image. Thus speckle reduction has become a very essential exercise for diagnoses. A wavelet-based image denoising technique is an effective filter to reduce speckle. Homomorphic processing is an approach for wavelet filtering. In this approach, to convert multiplicative noise into additive noise, first logarithmic transform is performed, and then wavelet filtering and exponential operation are performed. In the non-homomorphic approach, wavelet filtering is applied directly to the speckled image without any log or exponential operation. The present study compares the non-homomorphic and the homomorphic wavelet filtering techniques for the speckled images. Quantitative and qualitative results demonstrate that the non-homomorphic technique has higher performance than the homomorphic technique.
\end{abstract}

Index Terms-Image denoising, speckle noise, ultrasonic imaging, wavelet filtering.

\section{INTRODUCTION}

Today, ultrasonography is widely used to view the human body due to its low cost, portability, safety and, real-time imaging [1]. Beside these advantages, the disadvantage of the ultrasonic imaging is the poor image quality, which is affected by speckle noise. Other types of coherent imagery such as synthetic aperture radar and laser-illuminated imagery have speckle noise [2]. When the ultrasonic beam is scattered by microscopic structure, speckle occurs in the image as a granular pattern [3]. Speckle noise reduces the image contrast and blurs image details, thus it is difficult to resolve normal and pathological tissue [4]. According to [5], [6], speckle complicates post-processing, such as edge detection, and decreases lesion detectability eight-fold. The target of speckle reduction is to remove the speckle while preserving as much as possible the important features of an image. Removing speckle noise in images is the major challenge in the study of medical imaging because the speckle noise is multiplicative noise. According to additive noise, removal of multiplicative noise is difficult without distorting the image details. For many discrete wavelet transform (DWT), the traditional approach is to use a logarithmic transform to convert multiplicative noise to additive noise [7]. After wavelet filtering, exponential operation is performed. However, these operations cause biased estimation of the signal and increase the

Manuscript received December 9, 2014; revised June 15, 2015

M. A. Gungor is with the Department of Electronics and Automation, Hitit University Vocational High School, 19169, Corum, Turkey (e-mail: alparslangungor@hitit.edu.tr).

I. Karagoz is with the Department of Electrical and Electronics Engineering, Faculty of Engineering, Gazi University, 06570, Ankara Turkey (e-mail: irfankaragoz@gazi.edu.tr). computational complexity [2]. This paper compares the non-homomorphic and the homomorphic wavelet filtering techniques for the speckled images.

\section{NON-HOMOMORPHIC AND HOMOMORPHIC DISCRETE WAVELET TRANSFORM}

DWT is used to view or process digital images at multiple resolutions. Fig. 1 shows DWT of an image [8].

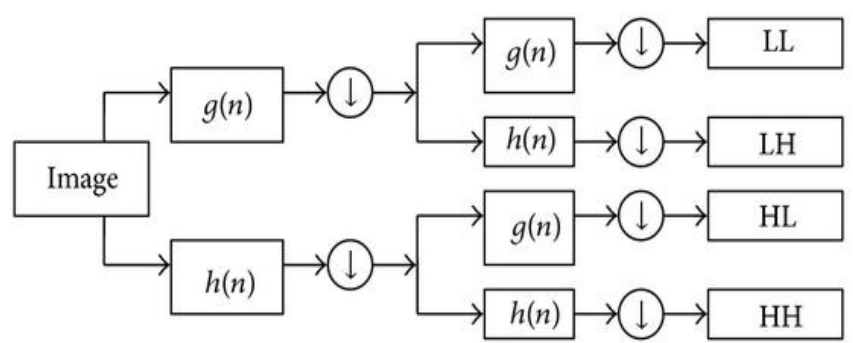

Fig. 1. DWT decomposition of an image.

In Fig. 1, after filtering the image with a pair of quadrature mirror filters along the rows and columns alternately, downsampling by a factor of two in each direction [9]. DWT decomposes the image and produces four subbands low-low (LL), low-high (LH), high-low (HL) and high-high (HH). LL coefficients are called approximation coefficients; LH, HL and $\mathrm{HH}$ coefficients are called horizontal, vertical and diagonal detail coefficients, respectively. The LL subband can further be decomposed to obtain another level of decomposition. For each level of the wavelet decomposition, four new images are created from the original image. As seen in [10], [11], downsampling may not be used to obtain an equal number of coefficients at each resolution scale but this increases the computational costs significantly and causes transmitting or storing the double of information with the decomposed signal.

Speckle noise corresponds to a high-frequency component of the image and appears in wavelet coefficients [12]. Thus wavelet filtering is widely used in denoising medical images. Homomorphic wavelet filtering technique involves five steps: 1) Take the logarithmic transform of the image; 2) Calculate DWT; 3) Process the wavelet coefficients; 4) Compute IDWT to obtain the reconstructed image; 5) Take the exponential of the reconstructed image. For non-homomorphic wavelet filtering technique, step 1 and step 5 are not used. For both homomorphic and non-homomorphic wavelet filtering techniques, how to process the wavelet coefficients is important. One widespread method to process the wavelet coefficients uses a thresholding operator. There are two different thresholding methods frequently used, hard thresholding and soft thresholding. 
Hard thresholding preserves the wavelet coefficients whose absolute values are larger than the threshold, otherwise they are set to zero

$$
h t(x)= \begin{cases}0, & |x|<T \\ x, & |x| \geq T\end{cases}
$$

where $x$ is image value and $T$ is threshold value.

In soft thresholding, wavelet coefficients whose absolute values are lower than the threshold are set to zero, otherwise this method shrinks them toward zero

$$
\operatorname{st}(x)= \begin{cases}0, & |x|<T \\ \operatorname{sign}(x) \cdot(|x|-T), & |x| \geq T\end{cases}
$$

Although soft thresholding eliminates the discontinuity that is inherent in hard thresholding, the efforts to find optimum thresholding method are underway [13].

Above-mentioned thresholding methods are applied to detail coefficients because the approximation coefficients contain the low frequency components. To find threshold value in equations (1) and (2), different rules were proposed in the literature. Donoho [14] proposed universal threshold defined by the following equation:

$$
T=\sigma \sqrt{2 \log N}
$$

where $N$ is the number of pixels in the image and $\sigma$ is noise standart deviation, determined by

$$
\sigma=\operatorname{median}\left(\left|Y_{i, j}\right|\right) / 0.6745
$$

where $Y_{i, j}$ represents the diagonal subband of first level wavelet decomposition of the image.

BayesShrink [15] is another method to calculate a threshold, determined by

$$
T=\sigma^{2} / \sigma_{X}
$$

where $\sigma_{X}$ is the image standart deviation evaluated in each wavelet subband. Instead of selecting an optimal threshold, a different method was proposed in the literature that detail coefficients are set to zero [16]. This method accepts that not only small values but also large values of detail coefficients have speckle noise.

\section{RESULTS AND DISCUSSION}

We used Lena, kidney and real ultrasound speckled images in the present work. Fig. 2(a) shows original Lena image. The MATLAB Image Processing Toolbox (IPT) function, imnoise, was used to add speckle noise to the original Lena image. Three noisy Lena images which have variances of 0.2 , $0.4,0.8$ were obtained. Fig. 2(b) shows a noisy Lena image with variance of 0.2. Second type of image we used in our study was the kidney image. We obtained the kidney image from the AnatQuest library (http://anatquest.nlm.nih.gov), as also seen in [17], [18]. This kidney image was used as the input image for Field II software [19], [20] to produce the noisy kidney image. The noise-free and noisy kidney images are shown Fig. 3(a) and Fig. 3(b), respectively. Finally, we used real ultrasound image of liver metastasis downloaded from an online ultrasound gallery www. ultrasound-images.com (Fig. 4(a)).

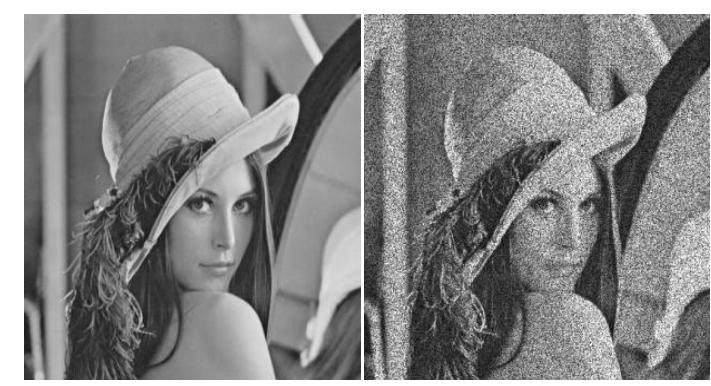

(a)

(b)

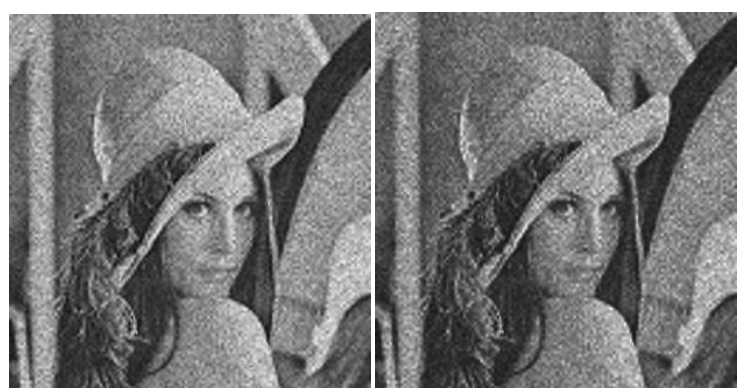

(c)

(d)

Fig. 2. (a) Original Lena image. (b) Noisy Lena image with variance of 0.2 . (c) Filtered Lena image with non-homomorphic approach. (d) Filtered Lena image with homomorphic approach.

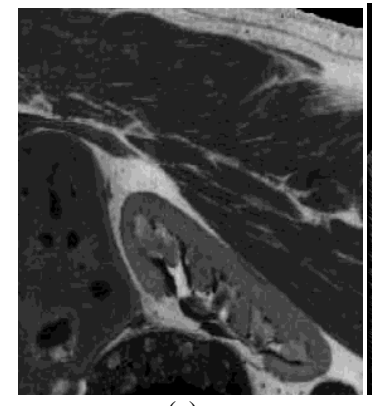

(a)

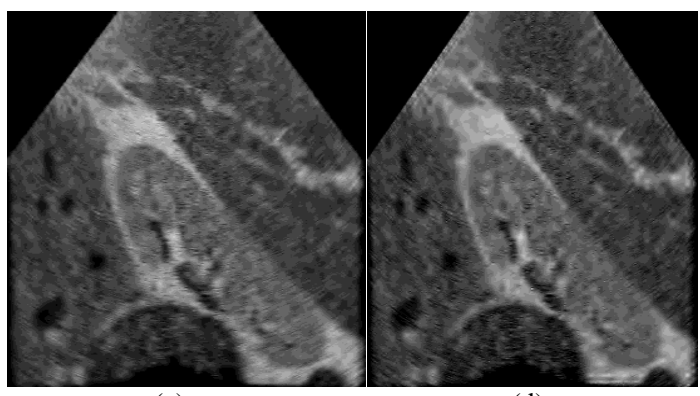

(c)

(b)

(d)

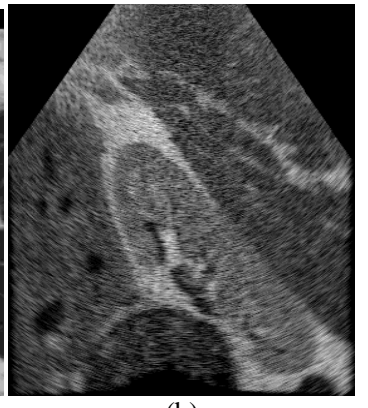

Fig. 3. (a) Kidney image retrieved by AnatQuest. (b) Noisy kidney image, generated by Field II. (c) Filtered kidney image with non-homomorphic approach. (d) Filtered kidney image with homomorphic approach.

Beta and PSNR metric were used to evaluate results. The beta metric [21] is defined by:

$$
\begin{aligned}
& \beta=\Gamma(\Delta I-\overline{\Delta I}, \hat{\Delta I}-\overline{\Delta \hat{I}}) / \sqrt{\Gamma(\Delta I-\overline{\Delta I}, \Delta I-\overline{\Delta I}) . \Gamma(\Delta \hat{I}-\overline{\Delta \hat{I}}, \Delta \hat{\Delta I}-\overline{\Delta \hat{I}})} ; \\
& \Gamma\left(I_{1}, I_{2}\right)=\sum_{(i, j) \in \mathrm{ROI}} I_{1}(i, j) I_{2}(i, j)
\end{aligned}
$$

where $\Delta I$ and $\Delta \hat{I}$ represent the high pass filtered version of original image $I(i, j)$ and its denoising version $\hat{I}(i, j)$ obtained with $3 \times 3$-pixels standard approximation of Laplacian operator. $\overline{\Delta I}$ and $\overline{\Delta I}$ are the mean intensities of $\Delta I$ and $\Delta \hat{I}$, respectively.

Additionally to the beta metric, another metric used in this paper is the Peak Signal-to-Noise Ratio: 


$$
\begin{gathered}
P S N R= \\
10 \cdot \log _{10}\left(L^{2} / \frac{1}{n \cdot m} \sum_{i=1}^{n} \sum_{j=1}^{m}\left[I_{r e f}(i, j)-I_{f}(i, j)\right]^{2}\right)
\end{gathered}
$$

where $I_{r e f}$ is the reference image, $I_{f}$ is the noisy or filtered image, $n \cdot m$ is the image size, $L$ is the maximum possible pixel value of the image.

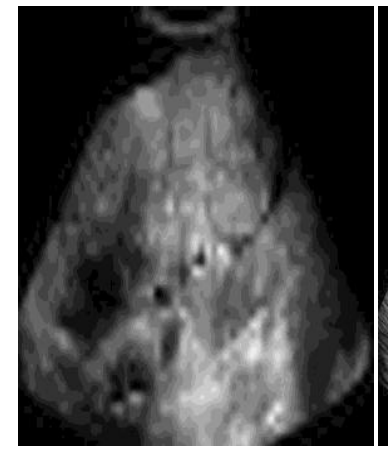

(a)

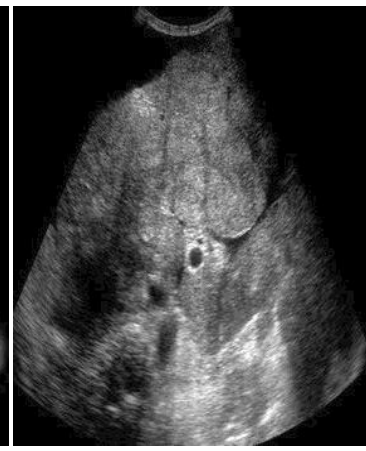

(b)

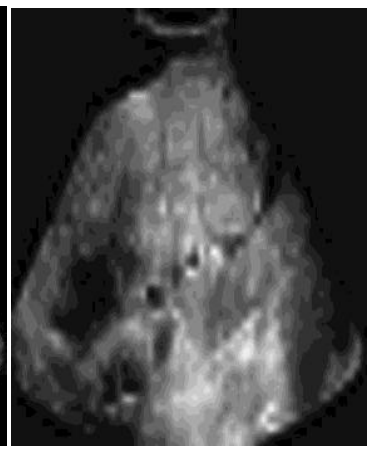

(c)

Fig. 4. (a) Real ultrasound image of a liver metastasis. (b) Filtered real ultrasound image with non-homomorphic approach. (c) Filtered real ultrasound image with homomorphic approach.

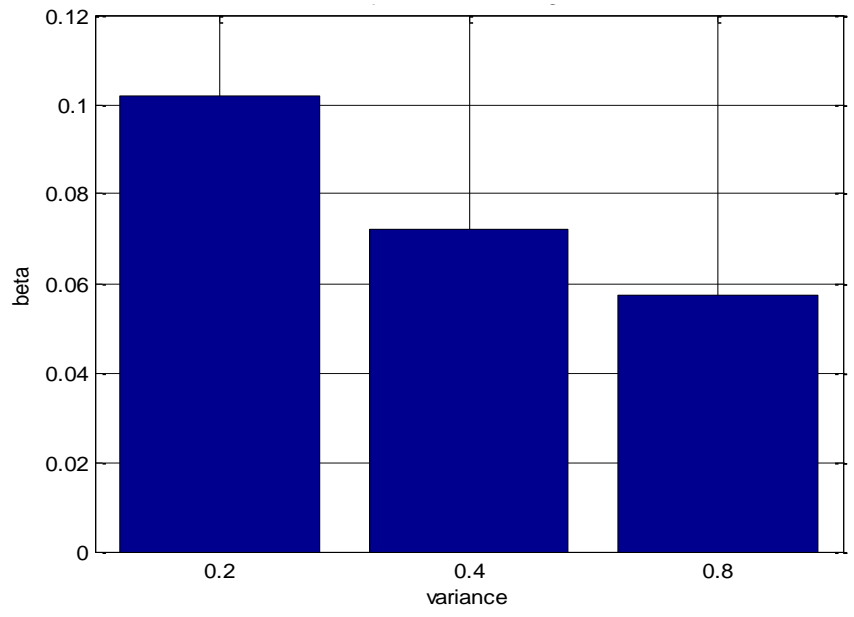

(a)

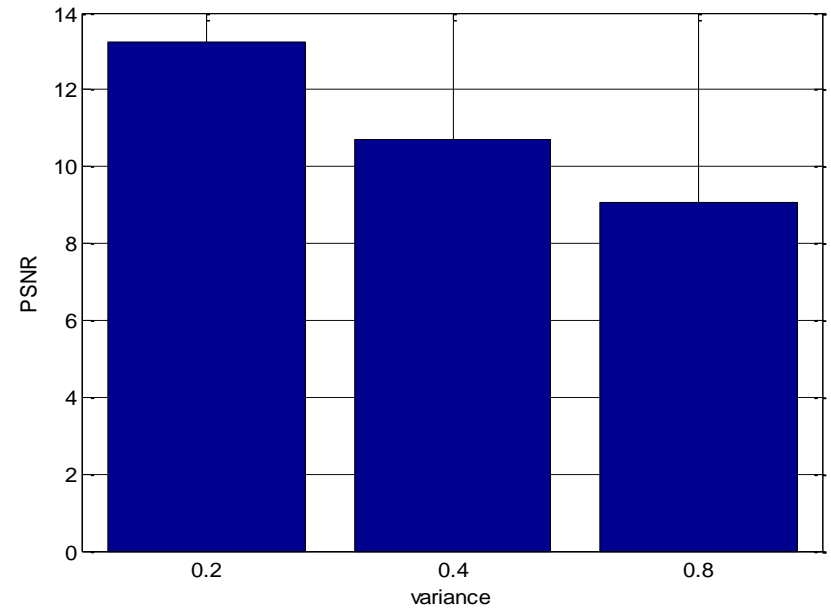

(b)

Fig. 5. (a) Beta. (b) PSNR vs. variance of noisy Lena image.

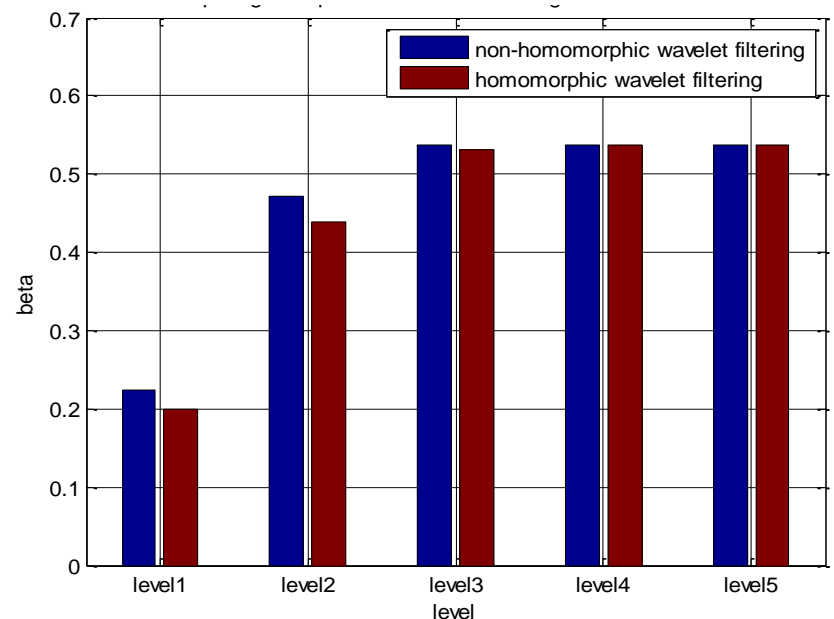

(a)

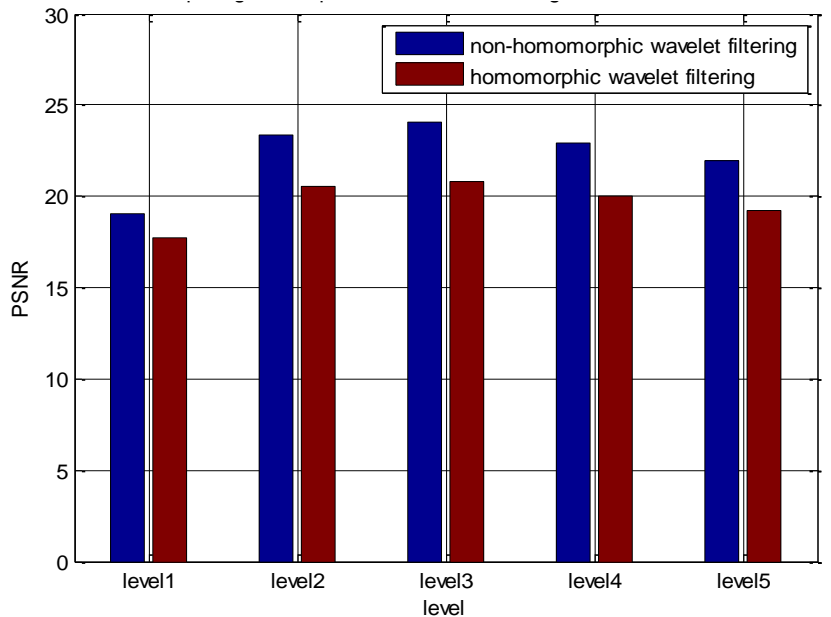

(b)
First of all, beta and PSNR were calculated for the noisy Lena images as function of the variance (Fig. 5). As can be seen both values decrease as noise variance increases. The non-homomorphic and the homomorphic wavelet filterings, based on soft thresholding, were applied for different levels. The results are shown in Fig. 6, Fig. 7 and Fig. 8 for Lena images with variance of $0.2,0.4$ and 0.8 , respectively. An increase in the decomposition until level 3 improves the results but the values beta and PSNR of level 4 and level 5 are same or slightly lower than that of level 3 for this study. The Fig. 2(c), Fig. 2(d), Fig. 6-Fig. 8) show that non-homomorphic wavelet filtering has better results than homomorphic wavelet filtering. Especially, for very noisy images, non-homomorphic filtering yields a more improved image than homomorphic filtering, as shown in Fig. 8.

Qualitative comparison was used to evaluate performance of filters for kidney and real ultrasound images because there are not reference images of these images. For the noisy 
kidney image, generated by Field II, the wavelet transform was performed with three-level wavelet decomposition. Filtered kidney image with non-homomorphic and homomorphic wavelet filterings are shown in Fig. 3(c) and Fig. 3(d), respectively. Finally, the detail coefficients of the real ultrasound image are set to zero for both non-homomorphic and homomorphic approaches. The result images are shown in Fig. 4(b) and Fig. 4(c). When all the filtered images of the noisy kidney and the real ultrasound images are analyzed, non-homomorphic approach has better

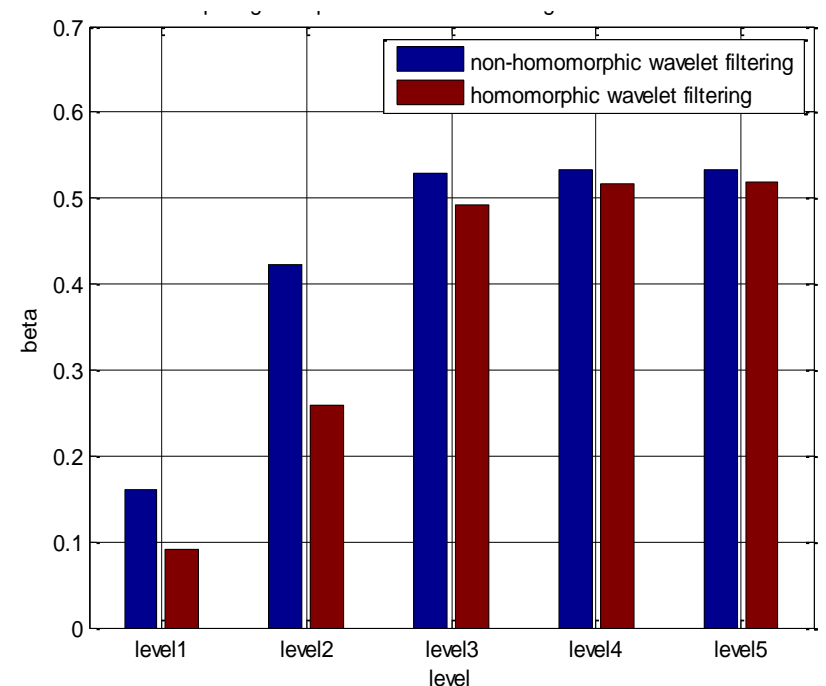

(a) results than homomorphic approach.

For homomorphic processing, logarithm of the image were taken but the images, used in this study, are dynamic range compressed images whose ranges are compressed for display. Then, log and exponential operations, used for homomorphic processing, cause biased estimation of the signal and increase the computational complexity so non-homomorphic processing gives better than homomorphic processing for dynamic range compressed images.

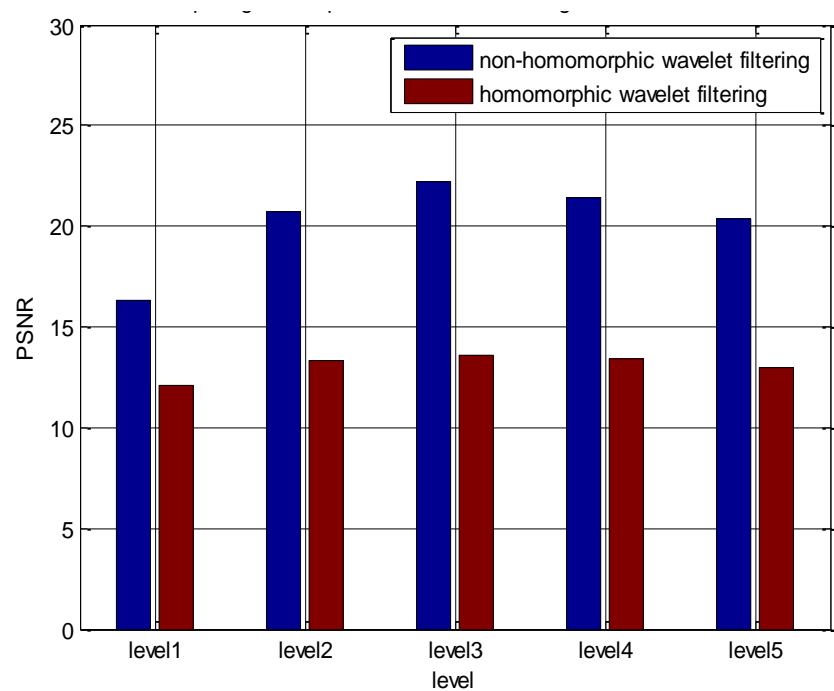

(b)

Fig. 7. (a) Beta. (b) PSNR vs. level of filtered Lena image with variance of 0.4.

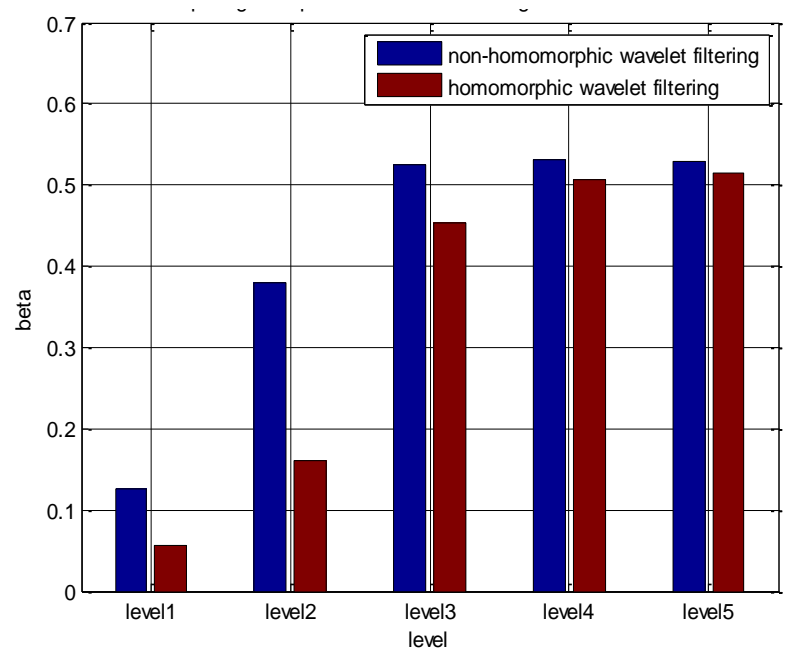

(a)

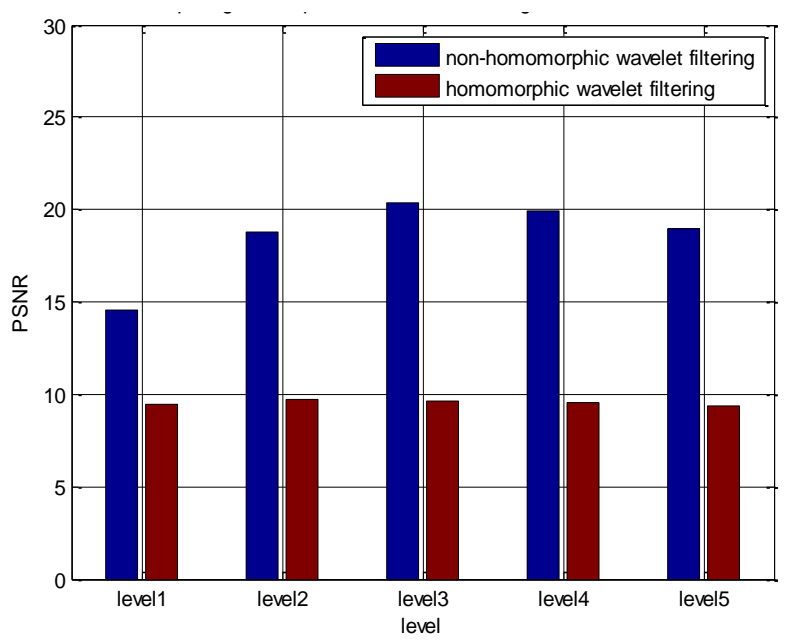

(b)

\section{CONCLUSION}

In this study, we compare the non-homomorphic and the homomorphic wavelet filtering techniques for the speckled image. We used the kidney image that is simulated by the Field II program, the real ultrasound image and the Lena image. Image quality was been evaluated using two different metrics (PSNR and beta) and qualitative comparison. For the kidney and Lena images, the detail coefficients were processed using soft thresholding and set to zero for real ultrasound image. The results obtained show us that the non-homomorphic wavelet filtering, applied directly without any $\log$ or exponential operation, provides better performance than the homomorphic wavelet filtering, applied with $\log$ and exponential operations, for the dynamic range compressed images.

\section{REFERENCES}

[1] T. L. Szabo, Diagnostic Ultrasound Imaging: Inside Out, United States of America: Elsevier Academic Press, 2004, ch. 1, pp. 23.

[2] S. Gupta, R. C. Chauhan, and S. C. Saxena, "Robust non-homomorphic approach for speckle reduction in medical ultrasound images," Medical and Biological Engineering and Computing, vol. 43, pp. 189-195, 2005 .

[3] A. Thakur and R. S. Anand, "Image quality based comparative evaluation of wavelet filters in ultrasound speckle reduction," Digital Signal Processing, vol. 15, pp. 455-465, 2005.

[4] M. Amirmazlaghani and H. Amindavar, "Wavelet domain Bayesian processor for speckle removal in medical ultrasound images," IET Image Process, vol. 6, no. 5, pp. 580-588, 2012. 
[5] C. Zhu, J. Ni, Y. Li, and G. Gu, "Speckle noise suppression techniques for ultrasound images," in Proc. 2009 Fourth International Conference on Internet Computing for Science and Engineering (ICICSE), 2009, pp. 122-125.

[6] J. C. Bamber and C. Daft, "Adaptive filtering for reduction of speckle in ultrasonic pulse-echo images," Ultrasonics, vol. 24, no. 1, pp. 41-44, 1986.

[7] A. Khare, M. Khare, Y. Jeong, H. Kim, and M. Jeon, "Despeckling of medical ultrasound images using Daubechies complex wavelet transform," Signal Processing, vol. 90, no. 2, pp. 428-439, 2010.

[8] R. C. Gonzalez, R. E. Woods, and S. L. Eddins, Digital Image Processing Using MATLAB, New Jersey: Pearson Education, Inc., 2004, ch. 7, p. 246

[9] S. G. Mallat, "A theory for multiresolution signal decomposition: The wavelet representation," IEEE Transactions on Pattern Analysis and Machine Intelligence, vol. 11, no. 7, pp. 674-693, 1989.

[10] A. Pizurica, W. Philips, I. Lemahieu, and M. Acheroy, "A versatile wavelet domain noise filtration technique for medical imaging," IEEE Transactions on Medical Imaging, vol. 22, no. 3, pp. 323-331, 2003.

[11] J. L. Starck, J. Fadili, and F. Murtagh, "The undecimated wavelet decomposition and its reconstruction," IEEE Transactions on Image Processing, vol. 16, no. 2, pp. 297-309, 2007.

[12] S. Sudha, G. R. Suresh, and R. Sukanesh, "Comparative study on speckle noise suppression techniques for ultrasound images," International Journal of Engineering and Technology, vol. 1, no. 1, pp. $57-62,2009$.

[13] G. Andria, F. Attivissimo, A. M. L. Lanzolla, and M. Savino, "A suitable threshold for speckle reduction in ultrasound images," IEEE Transactions on Instrumentation and Measurement, vol. 62, no. 8, pp. 2270-2279, 2013.

[14] D. L. Donoho and I. M. Johnstone, "Ideal spatial adaptation via wavelet shrinkage," Biometrika, vol. 81, no. 3, pp. 425-455, 1994.

[15] S. G. Chang, B. Yu, and M. Vetterli, "Adaptive wavelet thresholding for image denoising and compression," IEEE Transactions on Image Processing, vol. 9, no. 9, pp. 1532-1546, 2000.

[16] J. M. Mateo and A. Fernández-Caballero, "Finding out general tendencies in speckle noise reduction in ultrasound images," Expert Systems with Applications, vol. 36, no. 4, pp. 7786-7797, 2009.

[17] G. Andria, F. Attivissimo, G. Cavone, N. Giaquinto, and A. M. L. Lanzolla, "Linear filtering of 2-D wavelet coefficients for denoising ultrasound medical images," Measurement, vol. 45, no. 7, pp. 1792-1800, 2012.
[18] F. Adamo, G. Andria, F. Attivissimo, A. M. L. Lanzolla, and M. Spadavecchia, "A comparative study on mother wavelet selection in ultrasound image denoising," Measurement, vol. 46, no. 8, pp 2447-2456, 2013.

[19] J. A. Jensen, "Field: A program for simulating ultrasound systems," Medical \& Biological Engineering \& Computing, vol. 34, no. 1, pp. 351-353, 1996.

[20] J. A. Jensen and N. B. Svendsen, "Calculation of pressure fields from arbitrarily shaped, apodized, and excited ultrasound transducers," IEEE Trans. Ultrason., Ferroelec., Freq. Contr., vol. 39, no. 2, pp. 262-267, 1992

[21] F. Sattar, L. Floreby, G. Salomonsson, and B. Lovstrom, "Image enhancement based on a nonlinear multiscale method," IEEE Transactions on Image Processing, vol. 6, no. 6, pp. 888-895, 1997.

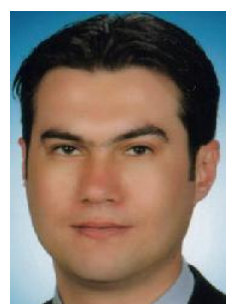

Murat Alparslan Gungor received his B.S. degree in electronics engineering from Istanbul University, Istanbul and the M.S. degree in electrical and electronics engineering from Gazi University, Ankara, Turkey, in 2000 and 2008, respectively.

$\mathrm{He}$ is currently a PhD. candidate at Gazi University, Department of Electrical and Electronics Engineering, Turkey. His research fields are signal processing, image processing and medical imaging systems.

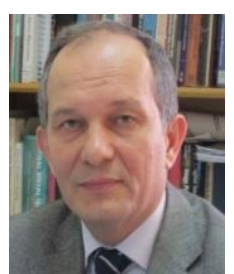

Irfan Karagoz received the B.S. and M.S. degrees in 1983 and 1985 from Bogazici University, Istanbul and Ph.D. degree in 1993 from Hacettepe University, Ankara, Turkey all in electrical and electronics engineering.

He became the professor in 2006. He established the Biomedical Calibration and Research Center in Gazi University and he became the manager of this center in 2005 . He held the same position and is the head of Electrical \& Electronics Engineering Department of Gazi University currently. His research fields are biomedical instrumentation, calibration of medical equipments, signal processing, speech processing and cochlear implants, image processing and medical imaging systems. 